\title{
Generation of highly confined optical bottle beams by exploiting the photonic nanojet effect
}

\author{
Myun-Sik Kim*a , Toralf Scharf ${ }^{\mathrm{a}}$, Stefan Mühlig ${ }^{\mathrm{b}}$, Carsten Rockstuhl ${ }^{\mathrm{b}}$, and Hans Peter Herzig ${ }^{\mathrm{a}}$ \\ ${ }^{a}$ Optics \& Photonics Technology Laboratory, Ecole Polytechnique Fédérale de Lausanne (EPFL), \\ Neuchâtel, CH-2000, Switzerland \\ ${ }^{\mathrm{b}}$ Institute of Condensed Matter Theory and Solid State Optics, Abbe Center of Photonics, Friedrich- \\ Schiller-Universität Jena, D-07743 Jena, Germany
}

\begin{abstract}
We report on the generation of photonic nanojets, which resemble optical bottle beams. They are realized by manipulating the illumination of dielectric microspheres. As illumination we use the outer region of deliberately truncated Bessel-Gauss beam or a focused Gaussian beam with intentionally induced spherical aberrations. For the Bessel-Gauss beam possessing a single side lobe only, the nanojet spot resembles an optical bottle beam with a strong confinement due to the nanojet effect. When multiple side lobes of the aberrated focal spot are used, a chain of 3D optical bottle beams appears. We show the $3 \mathrm{D}$ intensity distributions close to the spot and discuss the main characteristics of such optical bottle beams.
\end{abstract}

Keywords: photonic nanojet, beam shaping, optical bottle beam, complex light

\section{INTRODUCTION}

Optical bottle beam is a special kind of structured optical light field in which a dark focus is surrounded by regions of higher intensity ${ }^{1}$. It is of particular interest to trap particles that might be repelled and pushed away from the regions of maximum intensity ${ }^{2}$. For instance, low refractive-index particles, such as air bubbles in liquid, and metallic particles at certain frequencies are typically repelled by the bright spot $^{3,4}$. Absorbing (i.e. non-transparent) particles in liquids and gaseous medium are also repelled and pushed away from intensity maxima ${ }^{5-7}$. Micrometer-size aerosols, protein crystals, biological cells, artificial nano- and micro-structures, such as semiconducting nanowires, nanotubes, nanoclusters, are typical absorbing particles ${ }^{7}$. In addition, a dark trap is more beneficial for some applications than a bright trap for a simple reason: the light may not interact with the trapped object. This is of great advantage while trapping photosensitive materials, such as biological cells, and for trapping neutral atoms ${ }^{8-10}$. First attempts to generate dark traps were made by using beams with a doughnut-shape intensity distribution, which possess a vortex in their core ${ }^{11}$. Since this approach is limited to a transverse confinement only, the optical bottle beam, i.e. a truly three-dimensional (3D) dark spot, is considered as the best candidate for 3D optical trapping of such particular objects.

A number of approaches have been carried out to generate optical bottle beams or a 3D chain of such beams by using diffractive optical elements, axicons, spherical lenses, and interference of two beams ${ }^{12-16}$. In these studies, the confinement of the dark focus was often weak, because the numerical aperture (NA) of the focusing system has been fairly low. This leads to spot sizes of the dark focus in the range of a few micrometers or even larger. In contrast, we propose in this study a simple method to produce highly confined optical bottle beams and even 3D chains of them in the longitudinal direction. To this end, we rely on the scattering of light by an isolated micro-size dielectric sphere that creates a high-intensity, narrow beam-width and elongated spot, on the shadow-side surface of the sphere. This is known as a photonic nanojet ${ }^{17-19}$. Among numerous numerical and experimental studies of photonic nanojets, Kim et al. demonstrated for the first time experimentally the feasibility to shape photonic nanojets by optimized illumination ${ }^{20}$. Such engineered photonic nanojets, when used in combination with particular illuminations, can resemble an optical bottle beam with a strongly reduced size of the dark spot; ranging down to the submicron scale. Such highly confined optical bottle beams can be applied to trap single particles or individual small clusters within its tiny dark volume. By combining other inspection and analysis methods, e.g. Raman spectroscopy ${ }^{21}$, interferometry ${ }^{22}$ or far-field vectorial polarimetry ${ }^{23}$, the properties of such tiny particles can be investigated while being trapped.

*myunsik.kim@epfl.ch; phone 41-32-718-3279; fax 41-32-718-3201; http://opt.epfl.ch/

Complex Light and Optical Forces VI, edited by Enrique J. Galvez, David L. Andrews, Jesper Glückstad Marat S. Soskin, Proc. of SPIE Vol. 8274, 82740U · ( 2012 SPIE · CCC code: 0277-786X/12/\$18 - doi: 10.1117/12.905459 
In this paper we experimentally study the feasibility to generate optical-bottle-beam-like photonic nanojets by applying two particular illumination schemes: a Bessel-Gauss beam and an aberrated focused Gaussian beam, whose transverse extension (i.e. the spot size) is controlled by the NA of the focusing lens.

\section{EXPERIMENTAL SETUP}

To monitor in situ the transverse amplitude and phase distributions of initial illuminating beams, we conduct experiments in a high-resolution interference microscope (HRIM). The HRIM also serves to record the light emerging from the microsphere (i.e. the photonic nanojet). For the observation with the highest resolution in air, the HRIM is equipped with a 100X / NA 0.9 dry objective (Leica Microsystems, HC PL FLUOTAR). The HRIM operates in transmission and can be considered as a modified Mach-Zehnder interferometer as shown in Fig. 1(a).

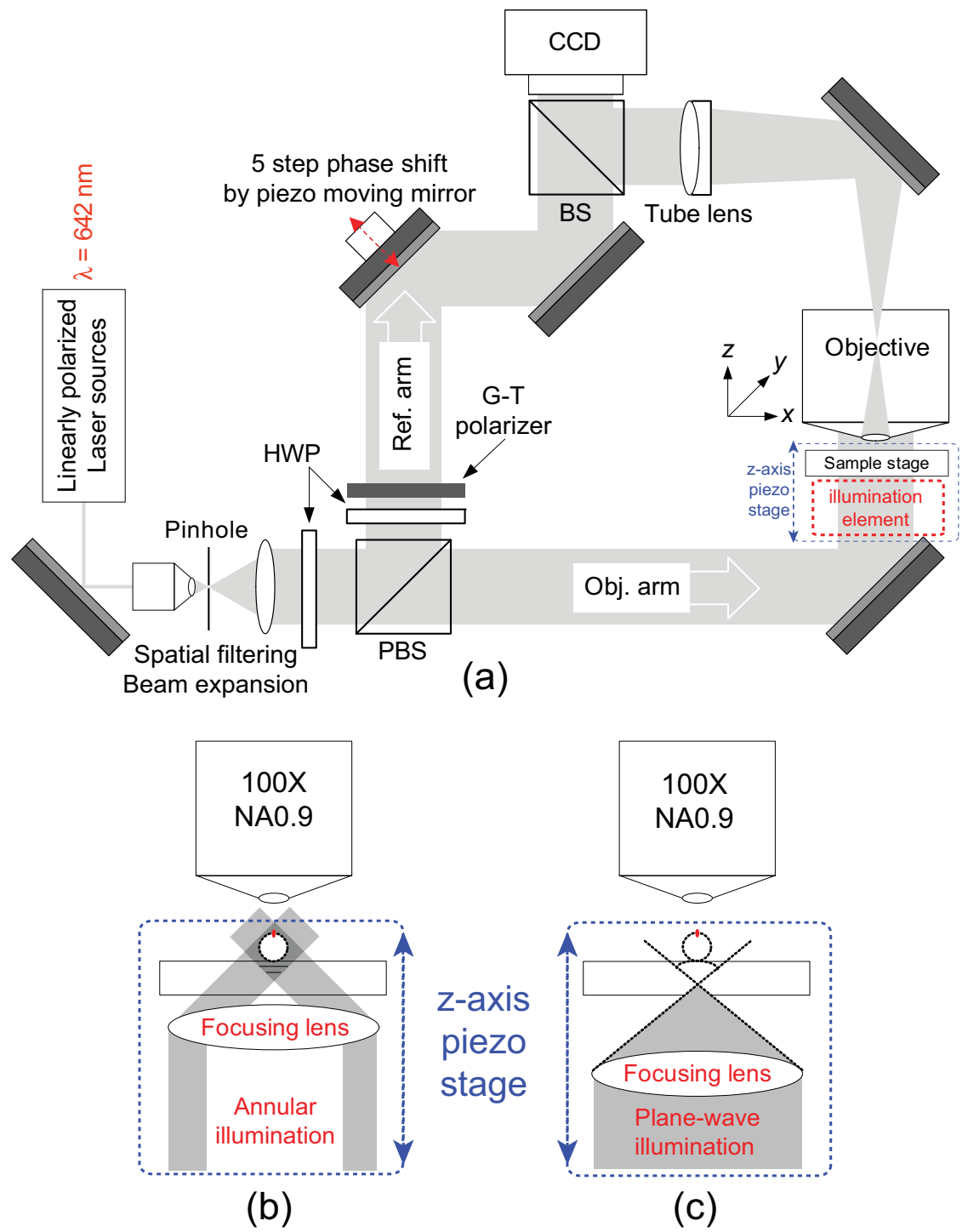

Figure 1. (a) Schematic view of the experimental setup. After divided by the polarizing beam splitter (PBS), a linearly polarized plane wave emerges in both the reference (Ref.) and object (Obj.) arms. In the reference arm, the piezo driven mirror modulates the optical path with five steps of $90^{\circ}(=\lambda / 4)$. In the object arm, additional illumination elements can be used to manipulate the incident illumination: (b) a focusing lens with an annular illumination and (c) a focusing lens with a plane-wave illumination. The focusing elements are placed on the same piezo stage with the sample in order to preserve the illumination conditions while scanning the light fields in 3D. 
A single mode polarized laser diode (CrystaLaser, $642 \mathrm{~nm}$ : DL640-050-3) is used as coherent light source. Its output beam is expanded and collimated by a spatial filtering technique to form a plane wave. This plane wave, which is normally incident upon the sample, propagates along the positive $z$-axis and is polarized in $x$-direction. In the classical interferometric arrangement a polarizing beam splitter (PBS) divides the beam in a reference and an object arm with adjustable energy ratio. Half wave plates (HWP) and Glan-Taylor (G-T) polarizers are used to adjust the intensities and to optimize the contrast of the interference fringes. In the reference arm, a piezo-electrically driven mirror is mounted to change the optical path length. The phase distribution of the wave field is obtained by measuring the interference fringes at different mirror positions and employing the well-known 5-frame algorithm, which is called Schwider-Hariharan method $^{24,25}$. Five frames of the intensity pattern, from which the 2D phase information can be directly retrieved, are recorded while each frame suffers from an additional optical path of $\lambda / 4$ or $0.5 \pi$. The sample is mounted on a precision piezo stage with a $z$-scan range of $500 \mu \mathrm{m}$ and a nominal accuracy of $1 \mathrm{~nm}$ (MAD LAB CITY, NANO Z500). This $z$-axis piezo stage is used to define precisely the plane of interest at the highest resolution and measure 3D light fields emerging from the wavelength-scale objects by scanning the sample along the optical axis.

Under standard measurement conditions, an expanded collimated beam which resembles a plane wave with linear polarization along the $x$-axis illuminates the sample as depicted in Fig. 1(a). By including additional illumination components, such as an aperture, a lens, or a spatial light modulator into the object arm before the object, the amplitude, the phase, or the polarization distribution of the incident beam can be modified. Figures 1(b) and 1(c) show the usage of the illumination elements for two particular illuminating beams, where the sphere is olny shown for completeness at this point. In such a way, a Bessel-Gauss beam can be generated by focusing an incident parallel annular beam [see Fig. 1(b)]. Aberrations can be induced in a focused Gaussian beam by slightly defocusing [see Fig. 1(c)]. Here, we define a reference plane, called the entrance plane of the sphere, as the plane where the illumination starts to interact with the sphere. This plane stands perpendicular to the optical axis and at a distance of a radius away from the sphere's center. A more detailed description of how the illumination can be engineered can be found in Ref. 20. When illumination components are inserted underneath the sample, they are fixed on the same piezo-stage as the sample. Since a planewave incidence on the illumination component is guaranteed, the sample together with illumination elements can be scanned in the axial (z) direction without changing the illumination conditions. In this manner, we assure to measure 3D amplitude and phase distributions with fixed and specific illumination conditions.

\section{BESSEL-GAUSS BEAM ILLUMINATION}

Bessel beam is a non-diffracting beam ${ }^{26,27}$, which propagates with a planar phase evolution in space and a radial amplitude distribution corresponding to a Bessel function. Each ring of the Bessel beam carries the same amount of energy which adds up to infinity. The phase among adjacent rings differs exactly by the value of $\pi$, and the Fourier spectrum of such Bessel beam is an infinitely thin ring. Therefore, perfect Bessel beams are inaccessible in a true experiment and their spatial distribution can only be sufficiently approximated within some finite distance. These approximations can be met experimentally while focusing the light transmitted through a sufficiently narrow ring upon plane wave illumination, as depicted in Fig. 1(b). Such an illumination will be used in our experiment. Figure 2 shows the transverse ( $x-y$ plane) intensity and phase distributions of two different Bessel-Gauss beams, which are generated by objectives with different numerical apertures but the same illumination. The wavefronts of such beams are planar but the transverse extension is not continuous, as seen in Figs. 2(b) and 2(d) where one observes the discrete wavefronts and their phase difference of $\pi$ in adjacent rings.

Our interest is to exploit such a discrete-wavefront illumination by employing only the central and $1^{\text {st }}$ side lobe for illumination. This area is indicated by a white dashed circle in Fig. 2. The size of the illumination (i.e. the lateral extension of the central and the $1^{\text {st }}$ lobes of the Bessel-Gauss beam) primarily depends on the NA of the focusing lens assuming that the obstruction ratio of the annular pupil is fixed. In our case the central obstruction is set to $70 \%$ of the entrance pupil. Generally, the focal spot that is observed with such illumination is slightly smaller than the focal spot found for a focused Gaussian beam of the same NA since the Bessel function leads to the narrower central lobe than the Airy function ${ }^{28,29}$. The FWHM size of the central lobe is measured to be $1.3 \mu \mathrm{m}$ for NA 0.22 and $4 \mu \mathrm{m}$ for NA 0.15 . In Fig. 2, the corresponding diameter of the illumination beam indicated by the white dashed circle is approximately $6 \mu \mathrm{m}$ and $15.5 \mu \mathrm{m}$, respectively. The illumination is designed to work with microspheres of $5-\mu \mathrm{m}$ and $12-\mu \mathrm{m}$ diameters. Camera images of the beam and the interaction with a sphere are shown in Fig. 3. 


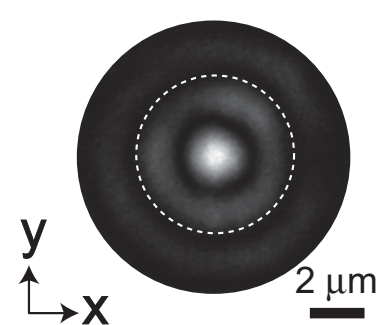

(a)

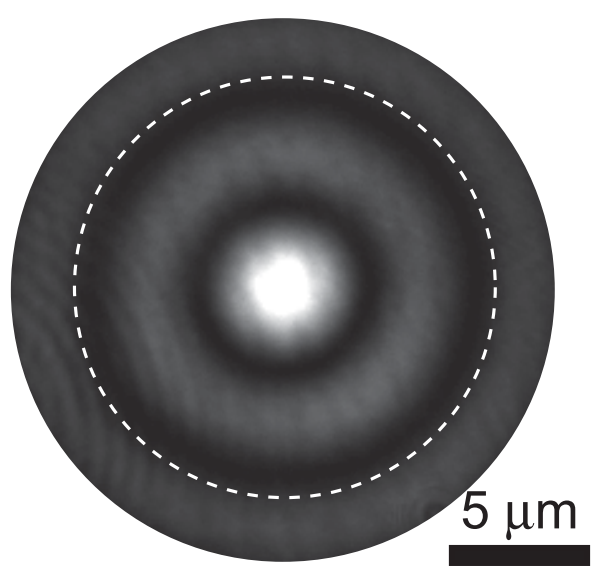

(c)

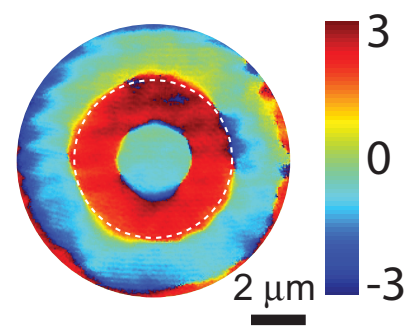

(b)

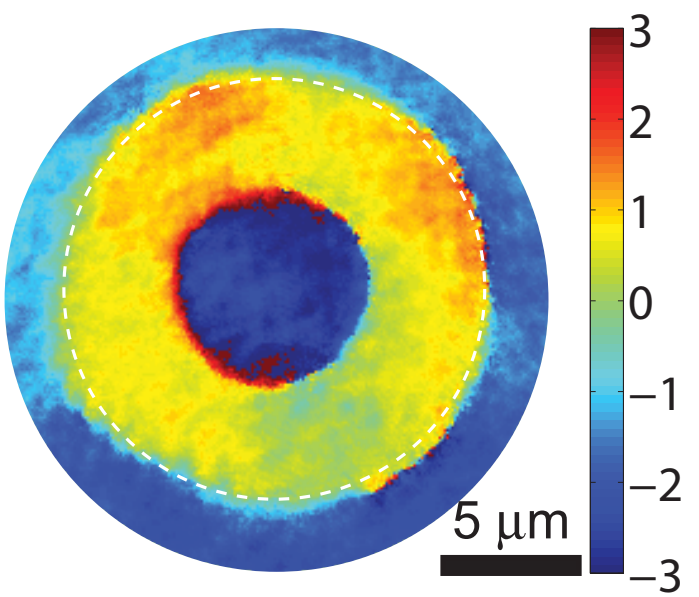

(d)

Figure 2. (Color online) The measured transverse $(x-y)$ intensity and phase distribution of Bessel-Gauss beams generated by objectives with different NAs: (a) intensity and (b) phase for NA 0.22 objective and (c) intensity and (d) phase for NA 0.15 objective. The white dashed circles indicate the central and the $1^{\text {st }}$ side lobes of both NAs, which are used for the illumination.

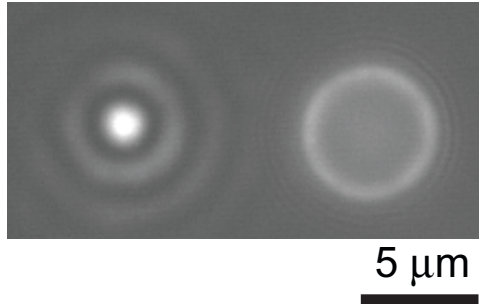

(a)

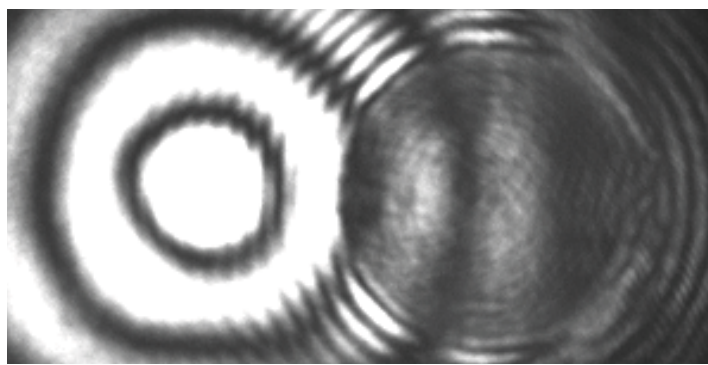

(b)
$5 \mu \mathrm{m}$

Figure 3. CCD images of the microsphere with the illumination beam aside: (a) 5- $\mu \mathrm{m}$ sphere with a Bessel-Gauss beam generated by the NA0.22 objective and (b) $12-\mu \mathrm{m}$ sphere with Bessel-Gauss beam by the NA0.15 objective.

Such illumination leads to particular photonic nanojets, which resemble the optical bottle beam. Figure 4 shows beams with a dark intensity focus, which is found directly after the primary bright photonic nanojet spot, surrounded in all directions by regions of higher intensity. These specific optical-bottle-beam-like photonic nanojets exhibit a submicronsize central dark focus for both experimental conditions given in Fig. 3. The size of the dark focus can be controlled by varying the operation wavelength, the sphere diameter, and/or the illuminating beam size. In order to obtain a submicron dark focus, one needs to work with sphere sizes smaller than approximately $20 \lambda$ (nanojet regime) at the visible spectrum and the illuminating beam (i.e. the central and the $1^{\text {st }}$ lobes of the Bessel-Gauss beam) has to have an analogous size. 


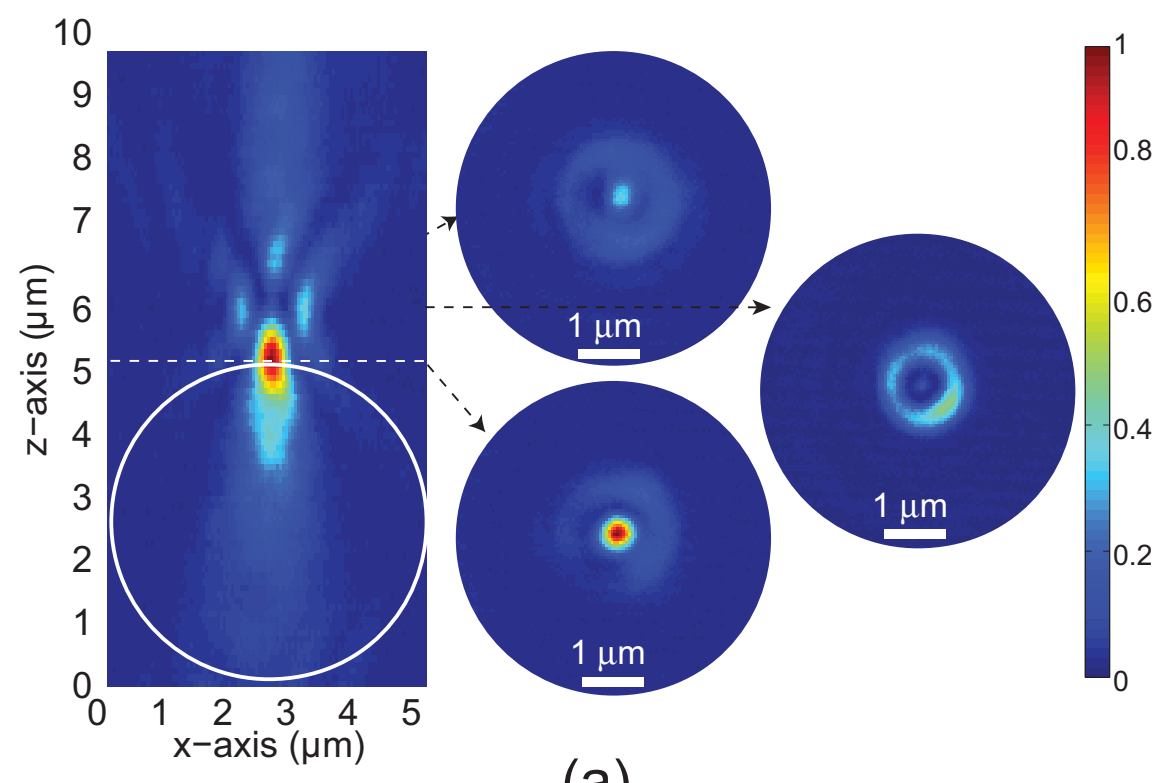

(a)

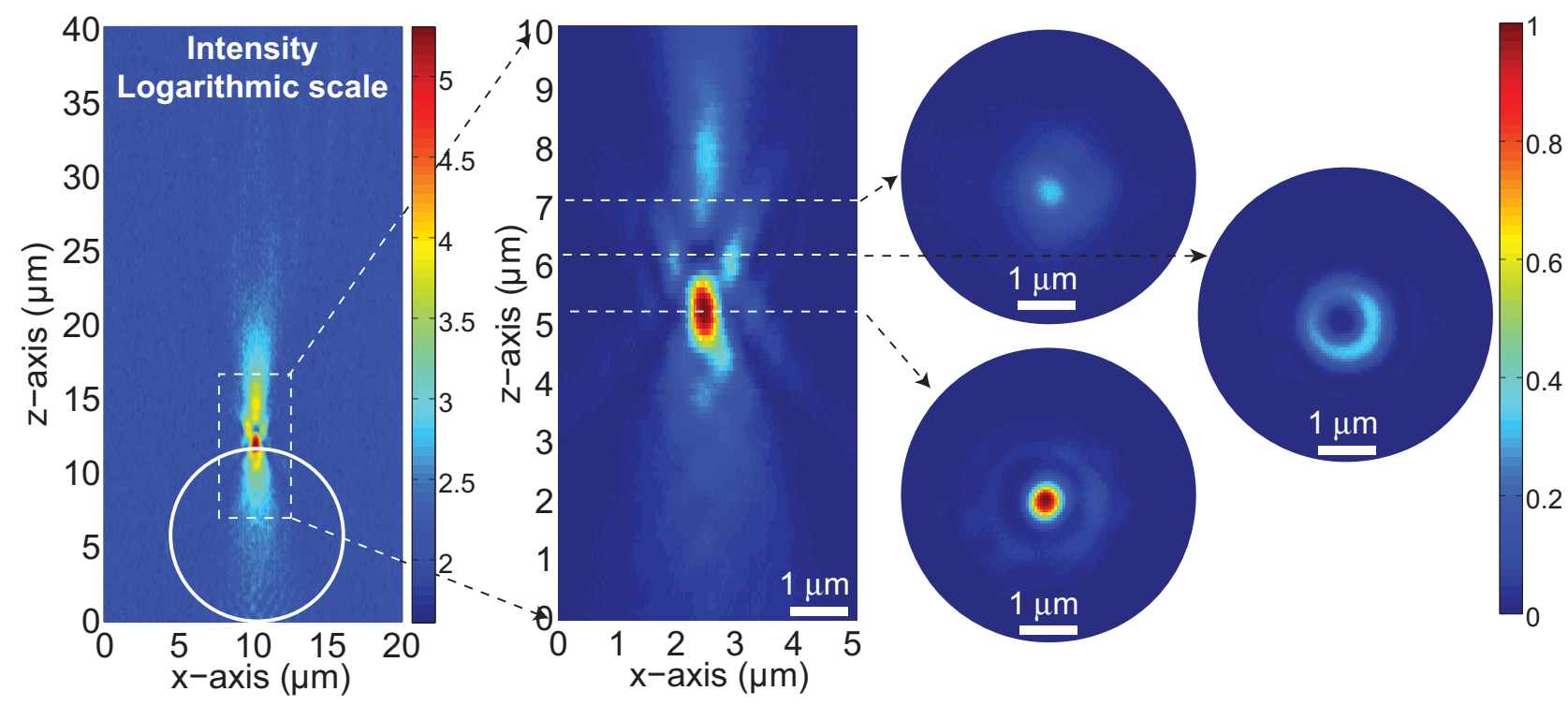

(b)

Figure 4. (Color online) The measured longitudinal $(x-z)$ and transverse $(x-y)$ intensity distributions of the opticalbottle-beam-like photonic nanojets: (a) by 5 - $\mu \mathrm{m}$ glass sphere [see Fig. 3(a)] and (b) $12-\mu \mathrm{m}$ glass sphere [see Fig. 3(b)]. The white circles in the longitudinal intensity maps indicate the corresponding microspheres. The intensities are all normalized except in the logarithmic-scale map.

\section{ABERRATED FOCUSED GAUSSIAN BEAM ILLUMINATION}

In order to create a chain of such bottle-beam-like nanojet spots, we use another specific illumination, i.e. an aberrated focused Gaussian beam. This illumination is formed by using a 50X / NA0.9 objective (Leica Microsystems, HXC PL APO, coverslip correction 0) with $170-\mu \mathrm{m}$ thick coverslip, schematically depicted in Fig. 1(c). In HRIM, we can precisely control the illuminating beam by monitoring the intensity distributions and recording phase distributions at 
each defocusing situation. The reference plane for such defocusing is the $z=0 \mu \mathrm{m}$ plane, where it is assumed to focus on the bottom of the sphere. As an example, Fig. 5(a) shows details of the illuminating beam when the sphere is not inserted in the beam path: the longitudinal $(x-z)$ and transverse $(x-y)$ intensity distributions together with the $x-y$ phase map at the reference plane $[z=0 \mu \mathrm{m}]$. When the 12- $\mu \mathrm{m}$ sphere is inserted as shown in Fig. 5(b), a chain of optical-bottle-beam-like photonic nanojets emerges several micrometers behind the surface of the sphere. The phase difference among the side lobes and their notable intensities, which are clearly seen in the transverse intensity and phase maps in Fig. 5(a), play an important role in the creation of such chain beams with interference phenomenon. The transverse slices of the 3D intensity map through the bright and dark spots shown in Fig. 5(b) demonstrate highly confined optical bottle beams, whose dark focus remains within submicron size.

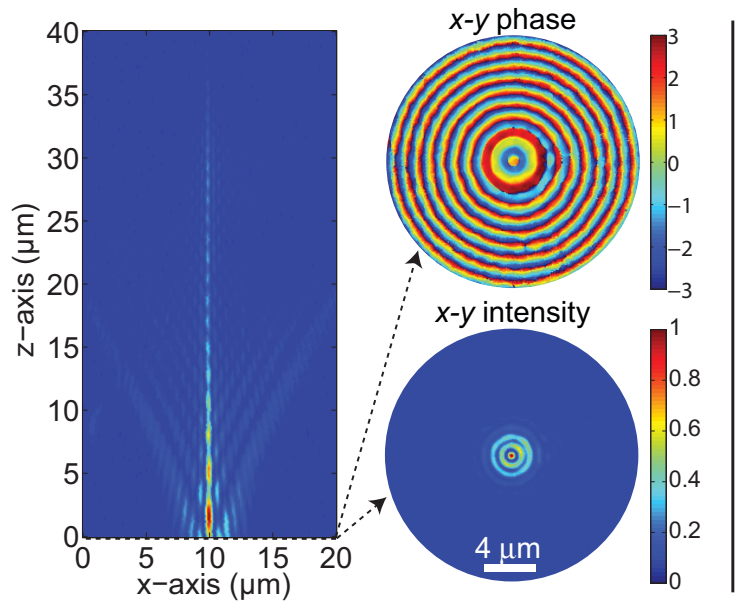

(a)
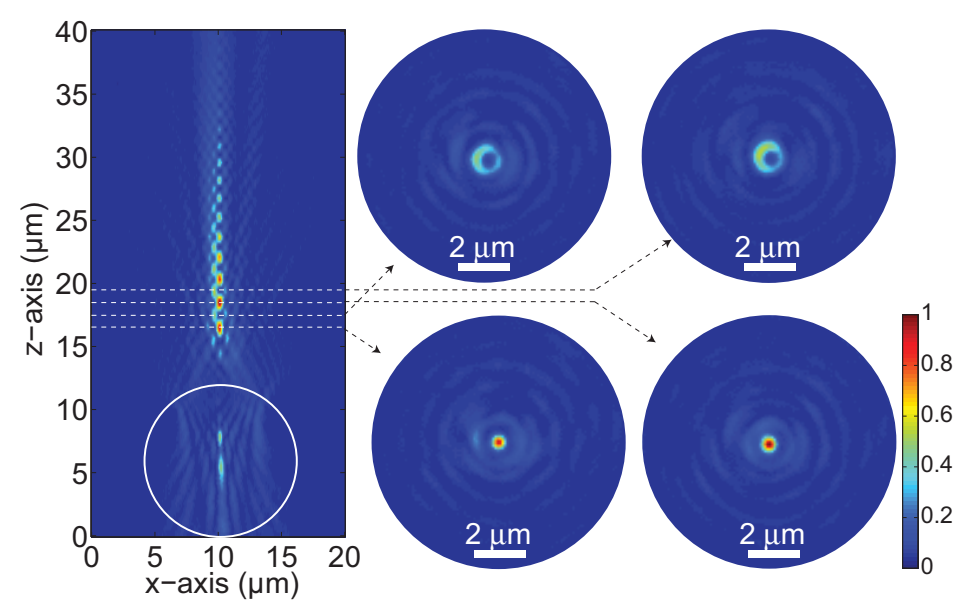

(b)

Figure 5. (Color online) (a) The measured longitudinal $(x-z)$ and transverse $(x-y)$ intensity distributions of the illumination beam without the sphere. The beam is intentionally aberrated and defocused. The $x-y$ phase at the reference plane, which is the $z=0 \mu \mathrm{m}$ plane where the bottom of the sphere sits, is shown, too. (b) The measured longitudinal $(x-z)$ and transverse $(x-y)$ intensity distributions of the chain of the optical-bottle-beam-like photonic nanojets, in which the white circle indicates the $12-\mu \mathrm{m}$ sphere. The intensities are all normalized.

It is reported in Ref. 20 that a diverging wavefront in general pushes the photonic nanojet away from the surface of the sphere. Moreover, the radius of curvature (ROC) of the wavefront governs the distance between the sphere surface and the nanojet spot because a smaller ROC leads to a larger distance. In our experiment for the bottle beam, although the wavefront at the reference plane is discrete, the overall curvature is diverging with defocusing of approximately $8 \lambda$. The diverging wavefront causes a shift of the focus and the first bright spot appears approximately $5 \mu \mathrm{m}$ behind the surface of the sphere. The size and number of chains of such beams can be adjusted by modifying the sphere size and the illuminating beam parameters, such as the relative intensities of each side lobe and the width of side lobes. These parameters are controllable by the NA of the objective, the thickness of the coverslip, and by defocusing. If trapping is considered, small objects are difficult to detach in adhesion to other objects or surface of the substrate due to adhesion forces. Therefore, such a larger working distance is advantageous for trapping and sorting applications because the risk to touch the sphere's surface is smaller.

\section{CONCLUSIONS}

We have investigated experimental feasibilities to generate highly confined optical bottle beams via the photonic nanojet phenomenon, in other words, the optical-bottle-beam-like photonic najojets. Standard photonic nanojets emerge on the rear side of dielectric microspheres under a plane-wave illumination. We applied particular illuminating beams, e.g., a Bessel-Gauss beam and an aberrated focused Gaussian beam, to microspheres of different diameters. A high-resolution interference microscope (HRIM) was employed to precisely control the illuminations and measure the resultant specific nanojet spots. For the Bessel-Gauss beam, the central and the 1st lobe were used to illuminate a microsphere with a size comparable to the lateral extension of the illumination. A submicron-size dark focus surrounded by higher intensity 
region in all directions is observed. At the same time, the brightest spot appears on the surface of the sphere just before the dark spot along the axial direction due to the photonic nanojet effect. When an aberrated focused Gaussian beam is slightly defocused from the entrance of the sphere (i.e. the reference plane), the side lobes possess relatively prominent intensity and their phases differ from each other. This specific illumination condition leads to form a chain of the opticalbottle-beam-like photonic najojets, in which the size of the dark focus is still maintained on a submicron scale. Such highly confined optical-bottle-beam-like photonic najojets can play important role in trapping and sorting of submicron objects or nanoparticles of which the bright trap is not applicable or less effective.

\section{ACKNOWLEDGMENT}

The research leading to these results has received funding from the European Community's Seventh Framework Programme FP7-ICT-2007-2 under grant agreement No. 224226.

\section{REFERENCES}

[1] Arlt, J. and Padgett, M. J., "Generation of a beam with a dark focus surrounded by regions of higher intensity: the optical bottle beam," Opt. Lett. 25, 191-193 (2000).

[2] Dienerowitz, M., Mazilu, M. and Dholakia, K., "Optical manipulation of nanoparticles: a review," J. Nanophoton. 2, 021875 (2008).

[3] Lankers, M., Popp, J., Urlaub, E., Stahl, H., Rössling, G. and Kiefer, W., "Investigations of multiple component systems by means of optical trapping and Raman spectroscopy,” J. Mol. Struct. 348, 265-268 (1995).

[4] Sasaki, K., Koshioka, M., Misawa, H., Kitamura, N. and Masuhara, H., "Optical trapping of a metal particle and a water droplet by a scanning laser beam,” Appl. Phys. Lett. 60, 807-809 (1992).

[5] Davis, E. J. and Schweiger, G., [The Airborne Microparticle: Its Physics, Chemistry, Optics, and Transport Phenomena], Springer, 780-785 (2002).

[6] Shvedov, V. G., Desyatnikov, A. S., Rode, A. V., Krolikowski, W. and Kivshar, Yu. S., "Optical guiding of absorbing nanoclusters in air," Opt. Express 17, 5743-5757 (2009).

[7] Shvedov, V. G., Hnatovsky, C., Rode, A. V. and Krolikowski, W., "Robust trapping and manipulation of airborne particles with a bottle beam," Opt. Express 19, 17350-17356 (2011).

[8] Chu, S., "The manipulation of neutral particles," Rev. Mod. Phys. 70, 685-706 (1998).

[9] Isenhower, L., Williams, W., Dally, A. and Saffman, M., "Atom trapping in an interferometrically generated bottle beam trap," Opt. Lett. 34, 1159-1161 (2009).

[10] Xu, P., He, X., Wang, J. and Zhan, M., "Trapping a single atom in a blue detuned optical bottle beam trap," Opt. Lett. 35, 2164-2166 (2010).

[11] Gahagan, K. T. and Swartzlander, Jr., G. A., “Optical vortex trapping of particles,” Opt. Lett. 21, 827-829 (1996).

[12] Garcia-Sucerquia, J., Medina, F. F. and Matteucci, G., "Optical tubular structures produced by diffraction of circular apertures," Opt. Lasers Eng. 42, 61-70 (2004).

[13] Chen, W. and Zhan, Q., "Three-dimensional focus shaping with cylindrical vector beams," Opt. Commun. 265, 411 417 (2006).

[14] De Angelis, M., Cacciapuoti, L., Pierattini, G. and Tino, G. M., "Axially symmetric hollow beams using refractive conical lenses," Opt. Lasers Eng. 39, 283-291 (2003).

[15] Wei, M.-D., Shiao, W.-L. and Lin, Y.-T., "Adjustable generation of bottle and hollow beams using an axicon," Opt. Commun. 248, 7-14 (2005).

[16]Philip, G. M. and Viswanathan, M. K., "Generation of tunable chain of three-dimensional optical bottle beams via focused multi-ring hollow Gaussian beam," J. Opt. Soc. Am. A 27, 2394-2401 (2010).

[17] Chen, Z., Taflove, A. and Backman, V., "Photonic Nanojet enhancement of backscattering of light by nanoparticles: a potential novel visible-light ultramicroscopy technique," Opt. Express 12, 1214-1220 (2004).

[18]Li, X., Chen, Z., Taflove, A. and Backman, V., "Optical analysis of nanoparticles via enhanced backscattering facilitated by 3-D photonic Nanojets," Opt. Express 13, 526-533 (2005).

[19] Heifetz, A., Kong, S.-C. , Sahakian, A. V., Taflove, A. and Backman, V., "Photonic Nanojets," J. Comput. Theor. Nanosci. 6, 1979-1992 (2009).

[20] Kim, M.-S., Scharf, T., Mühlig, S., Rockstuhl, C. and Herzig, H. P., "Engineering photonic nanojets," Opt. Express 19, 10206-10220 (2011). 
[21] Svedberg, F., Li, Z., Xu, H. and Käll, M., "Creating hot nanoparticle pairs for surfaceenhanced Raman spectroscopy through optical manipulation," Nano Lett. 6, 2639-2641 (2006).

[22] Hwang, J. and Moerner, W. E., "Interferometry of a single nanoparticle using the Gouy phase of a focused laser beam," Opt. Commun. 280, 487-491 (2007).

[23] Rodríguez-Herrera, O. G., Lara, D and Dainty, C., "Far-field polarization-based sensitivity to sub-resolution displacements of a sub-resolution scatterer in tightly focused fields," Opt. Express 18, 5609-5628 (2010).

[24] Schwider, J., Burow, R., Elssner, K.-E., Grzanna, J., Spolaczyk, R. and Merkel, K., "Digital wave-front measuring interferometry: some systematic error sources," Appl. Opt. 22, 3421-3432 (1983).

[25] Hariharan, P., Oreb, B. F. and Eiju, T., "Digital phase-shifting interferometry: a simple error-compensating phase calculation algorithm," Appl. Opt. 26, 2504-2506 (1987).

[26] Durnin, J., Miceli, J. J. and Eberly, J. H., "Diffraction-free beams," Phys. Rev. Lett. 58, 1499-1501 (1987).

[27] Indebetouw, G., "Nondiffracting optical-fields - some remarks on their analysis and synthesis," J. Opt. Soc. Am. A 6, 150-152 (1989).

[28] Born, M. and Wolf, E., [Principles of Optics, $7^{\text {th }}$ ed.], Cambridge University Press, Chap. 8, p. 412 (1999).

[29] Singer, W., Totzeck, M. and Gross, H., [Handbook of Optical Systems], Wiley, Vol. 2, p. 410 (2005). 